\title{
In Thought, Word, and Deed: Suicidal Behaviors of Adjudicated Youth
}

\author{
Kevin Corcoran, PhD, JD \\ Thomas Crofoot Graham, PhD
}

\begin{abstract}
This study examines suicide ideation, plans, and attempts by youth in two juvenile justice settings: incarcerated youth $(n=83)$ and youth with community service sentences $(n=$ 144). The incarcerated sample reported $51 \%$ suicide ideation while the community sample reported $22 \%$. For both samples, suicide contemplators were quite likely (60-70\%) to have a suicide plan, while about $35 \%$ reported a suicide attempt in the past 12 months. Contemplators were distinguishable from noncontemplators on scores for external problems of delinquency and aggression, internal problems of anxiety, depression, withdrawal and somatic complaints, general depression, and general health status. The results have implications for assessment, triage, and brief treatment in juvenile justice settings. [Brief Treatment and Crisis Intervention 2: 233-239 (2002)]
\end{abstract}

KEY WORDS: suicide, suicide ideation, adjudicated youths, juvenile justice, external problems, mental health services, assessment, diagnosis, depression, attention deficit hyperactivity disorder, conduct disorders.

The past 2 decades have seen a combination of social forces contributing to the current mental health status of youth in corrections systems. Suicide rates for adolescents in general were unacceptably high and tending to increase. During the last half-century suicide rates for adolescents underwent a dramatic increase nearly tripling from a rate of 4.5 per 100,000 in 1950 to 12 per 100,000 in 1996 (Jobes, Berman, \& Martin, 2000).

From the Graduate School of Social Work at Portland State University.

Contact author: Kevin Corcoran, PhD, JD, Professor, Graduate School of Social Work, University Center Building, Portland State University, Portland, OR 97207. E-mail: corcorank@pdx.edu.

(c) 2002 Oxford University Press
Suicide is the third leading cause of death due to injury for 13- to 19-year-olds (MacKay, Fingerhut, \& Duran, 2000).

The alarming rate of suicide and a dawning awareness of the need for mental health services by youth in corrections beginning in 1980 have increased recognition of the need for assessment and treatment of suicide and other mental health problems of youth (Hutchinson, 1993). At the same time, reviews of social service systems indicated that prevention and remedial services that might keep youth out of corrections have been declining. The result is that the most vulnerable youth (i.e., the poor, youth of color, and young women) are more likely to be sent to prison or other secured settings instead 
of a less restrictive, mental health treatment facility (Hutchinson, 1993; McCroskey, 1988; Prescott, 1997; Stehno, 1982).

Mental health treatment need, in fact, may have even an adverse impact on a youth in the public system. When identified as needing services these youth are likely to be transferred from one public service system to anotherfrom child welfare to mental health and juvenile corrections - without receiving the needed help (Litrownik, Taussig, Landsverk, \& Garland, 1999; Prescott, 1997). They seem to fall through the cracks as they are passed along the way of the social service system until they age-out or have adult sentences in state or federal institutions.

Incarcerated youth are likely to have a number of mental health diagnoses, including those most commonly associated with criminal behavior, such as conduct disorders, oppositional defiant disorder, and impulse control disorders (Hutchinson, 1993), and depression with major depression, in particular, diagnosed for as many as $20 \%$ of youth in custody. These youth may show signs of depression through anger, hostile feelings, and aggressive belligerent behaviors along with symptoms of withdrawal or sadness. Suicidal gestures, thoughts, and suicide attempts accompany major depression and are common for youth in juvenile corrections (Hutchinson, 1993).

A reasonable approach to these problems includes a de-emphasis on juvenile justice solutions and emphasizes prevention, assessment, and remedial services. The first step would include a mental health assessment for all youth entering the juvenile justice system, a recommendation averred in Oregon (Office of Mental Health Services, 1996). For youth who enter the corrections system there is a need for increased awareness of suicide. A careful screening followed by a thorough assessment is recommended and should include a suicide assessment survey administered to every youth taken into custody (Hutchinson, 1993). Vital components of a suicide assessment include a physical examination, a mental status examination, and a family and social history sensitive to physical, emotional, or sexual abuse (Hutchinson, 1993). Specific questions for issues relevant to adolescent girls and boys alike should include histories of violence, self injury, head injury, and pregnancy - including impregnating a woman, sexual assault, and sexually transmitted disease (Prescott, 1997). The assessment should include factors unique to corrections settings, such as sexual assaults or abuse of authority by an officer. Once a suicide risk is identified, a determination of the youth's "reasons for living" (Osman et al., 1998; in Corcoran \& Fischer, 2000) greatly facilitates goals of brief treatment for suicidal youth.

Given the extent of the problem, the literature and research base is minimal and needs additional data. Further research is needed on mental health status and suicide assessment of youth who are most vulnerable, most likely to receive unequal treatment, most likely to have multiple problems, and very likely to be in settings that do not understand or meet their needs. The purposes of this study, then, are to describe suicidal behavior in two juvenile justice settings, as related to mental health history, self-identified need, and the associated mental health and behavior problems. The findings are informative for assessment, triage, and crisis intervention for youth at risk of self-harm.

\section{Methodology}

\section{Participants and Procedures}

Data were collected from two samples: adjudicated youth sentenced to community service and incarcerated youth. The community service sample consisted of 144 youth from three counties in Oregon, a rural county in the high desert of eastern Oregon, a county in the coastal range, and a large metropolitan location. There were no 
statistically significant differences between the three locations on any of the variables in questions, except for having seen a mental health professional where urban youth report less use than the rural counties $\left(\chi^{2}=4.96, p<.05\right)$. All participants were volunteers, recruited by their juvenile court counselor or probation officer. In consideration of completing a package of instruments, the youth were remunerated $\$ 10$ or credited with 1 hour towards their community service sentence. The instruments took approximately 30 minutes to complete and the testing period was credited towards the youngster's community service sentence.

The average age of the community service sample was 15.3 years, with a standard deviation of 1.5 . There were 31 females $(21.8 \%)$ and 111 males $(78.2 \%)$, and two participants did not complete the item.

The incarcerated sample were 83 youth from a medium-size institution holding a maximum of 97 inmates. The secured facility was constructed to accommodate the increased census due to a state mandatory sentencing law for loosely defined "violent" crimes; that is, crimes involving a weapon, which is any object used with the intent to harm, from a gun or knife to throwing a pencil across the classroom. Incarcerated youth were remunerated $\$ 2$ for completing the questionnaires, and the funds were allocated to a special correction center activity. The remuneration did not go directly to the youth as the facility prohibited inmates from possessing anything of value, especially money.

\section{Instrumentation}

The measurement package ascertained demographic variables and self-reported utilization of mental health services. Mental health service utilization included assessment services, treatment, and self-identified need. Assessment service use was defined by reporting that one had been referred to "a mental health professional to find out if you had a problem." Mental health service use included having "ever seen a counselor (e.g., a psychologist, social worker, or psychiatrist) for a personal problem" (i.e., individual mental health treatment) and having "ever seen a counselor for a family problem" (i.e., family treatment). Mental health service need was defined by whether or not the youth thought he or she currently needed to talk to a mental health professional about a problem (i.e., service need).

The measurement package also included assessments of general health status and depression, both from the SF-36 (Ware, Kosinski, \& Keller, 1995). Each is a single item index answered on a scale from one to five. While indices, the assessments have very good validity and utility. Mental health conditions or problems were also defined by the Child Behavior Checklist, Youth Self-Report version (CBCL; Achenbach, 1991). Problems were operationalized by scores on the externality subscale, which includes acting-out behaviors of delinquency and aggression, and the internality subscale, which consists of emotional withdrawal, somatic complaints, and anxiety and depression. Like the items from the SF-36, the CBCL is widely used and generally considered reliable and valid.

Suicidal behaviors were defined by three items: whether or not the youngster considered attempting suicide in the past 12 months; if so, whether they actually made a plan to commit suicide in the past 12 months; and if during the past 12 months the youth actually attempted suicide.

\section{Results}

\section{Suicidal Behaviors}

For the total sample, $32 \%$ reported having seriously considered suicide in the past 12 months. The rates of suicide contemplators were differ- 
ent for incarcerated youth $(51 \%)$ compared to youth with community service sentences $(22 \%)$, which were significantly different, $\chi^{2}(1)=19.5$, $p<.01$. Of the community service sample who had considered suicide, $61 \%$ reported having developed a suicide plan, while $71 \%$ of the incarcerated suicide contemplators reported a suicide plan. These proportions were not significantly different $\left(\chi^{2}<1.0, \mathrm{~ns}\right)$.

While the majority of contemplators reported a suicide plan, most did not report a suicide attempt (i.e., 62 and $65 \%$ for the incarcerated and community service samples, respectively). This should still be considering alarming that over one out of three youths in the juvenile justice system reported having made a suicide attempt within the past year.

\section{Mental Health Service History and Self-Reported Need}

Youth who have seriously considered suicide were more likely to have been referred for mental health services than noncontemplators $\left(\chi^{2}(1)=\right.$ $17.8, p<.01)$, but were not more likely to follow through and actually see a mental health professional $\left(\chi^{2}(1)=2.5\right.$, ns). Contemplators and noncontemplators were different on the proportion indicating a need or no need to talk to a mental health professional about a personal problem, $\chi^{2}$ (1) 26,8, $p<.01$; however, this difference seems due to the low rate of endorsing the need for mental health services by youth who have not considered suicide (i.e., 14\%), while contemplators were equally as likely to say they needed to see a mental health professional (47\%) as they were to say they did not need to see one $(53 \%)$.

\section{Association of Behavior Problems, Health Indicators, and Suicide}

The internality and externality subscales of the CBCL were used to measure emotional problems and acting out problems. A single item index of depression and one to ascertain general health status index were also used. The internal problems subscale correlated $-.58, p<.01$, with the depression index and $-.17, p<.05$ with the general health status index. External problems scores correlated -.47, $p<.01$, and $-.15, p<.05$, with depression and general health, respectively. Internal and external problem scores were correlated .59, $p<.01$, as were health and depression, $-.17, p<.05$. These findings suggest that both subscales of the CBCL and depression are fairly similar with more than $22 \%$ common variance. Health, in contrast, seems rather independent, having less than $3 \%$ variance common to external and internal problems and depression.

As displayed in Table 1, suicide contemplators were significantly more likely to have lower health status, report more depression, and have more external and internal problems. These results were the same for incarcerated youth and youths serving community sentences, although incarcerated youth had higher externality scores, internality scores and depression scores and lower health scores $(26.8,20.7,2.4$, and 3.7, respectively) compared to the community service sample (18.7, 14.1, 3.7, and 4.8, respectively); a $t$ test $>4.1, p<.001)$.

\section{Predicting Suicide Intent}

A critical component for working with suicidal youth, in the justice system or elsewhere, is predicting self-harm. Such information is crucial for assessment, triage, and brief treatment. The current data provide information on predicting suicide two ways. First, with the strong association between those who report suicidal ideation and, in fact, report having developed a suicide plan. Simply asking the youth if he or she is suicidal will point to a majority who have developed a plan.

These data are also useful in predicting sui- 
TABLE 1. Comparison of Suicide Contemplators with Noncontemplators

\begin{tabular}{|c|c|c|c|c|c|}
\hline \multirow[b]{2}{*}{ Dependent variable } & \multicolumn{2}{|c|}{ Suicide contemplators } & \multicolumn{2}{|c|}{ Noncontemplators } & \multirow[b]{2}{*}{$t$-test } \\
\hline & $M$ & $S D$ & $M$ & $S D$ & \\
\hline \multicolumn{6}{|c|}{ Incarcerated youth sample } \\
\hline Depression & 3.1 & 1.6 & 4.2 & 1.4 & $3.2^{*}$ \\
\hline Externality & 31.6 & 11.8 & 21.3 & 9.8 & $-4.0^{*}$ \\
\hline Internality & 28.1 & 11.9 & 12.5 & 9.2 & $-6.2^{*}$ \\
\hline Health & 2.8 & 1.0 & 2.1 & 1.0 & $-3.1^{*}$ \\
\hline \multicolumn{6}{|c|}{ Community service sample } \\
\hline Depression & 4.1 & 1.4 & 4.9 & 1.1 & $3.7^{*}$ \\
\hline Externality & 25.4 & 10.8 & 16.9 & 9.7 & $-4.2^{*}$ \\
\hline Internality & 22.3 & 10.4 & 12.0 & 9.1 & $-5.4^{*}$ \\
\hline Health & 3.8 & 1.0 & 3.2 & 1.1 & $-2.7^{*}$ \\
\hline
\end{tabular}

TABLE 2. Predicting Suicide Ideation Status

\begin{tabular}{|c|c|c|c|c|c|c|}
\hline \multirow[b]{2}{*}{ Predictor variables } & \multicolumn{3}{|c|}{ Suicide ideation status } & \multicolumn{3}{|c|}{ Stepwise regression $\mathrm{R} \&$ (\% shared var.) } \\
\hline & $R$ & $R^{2}$ & $t$-test & Step $1(26 \%)$ & Step $2(28.9 \%)$ & Step $3(30 \%)$ \\
\hline Site & .30 & $9 \%$ & $4.6^{\star *}$ & & & .13 \\
\hline Externality & .43 & $19 \%$ & $6.9^{\star *}$ & & .21 & 17 \\
\hline Internality & .53 & $28 \%$ & $9.1^{* *}$ & .52 & .39 & .38 \\
\hline Depression & .40 & $16 \%$ & $-6.3^{* *}$ & & & \\
\hline Health & .14 & $2 \%$ & $-2.1^{*}$ & & & \\
\hline
\end{tabular}

${ }^{*} p<.05,{ }^{* *} p<.01$.

cide ideation status. Juvenile justice setting, external problems, internal problems, depression, and health status were all significantly related to suicide status, as displayed in Table 2. Stepwise regression analysis suggests that knowing a youth's internal problems of anxiety, depression, somatic complaints, and emotional withdrawal accounts for $26 \%$ of the variance. About 3\% additional variance is added by knowing a youth's external problems of delinquency and aggression. And finally, about $1 \%$ more variance is accounted for by whether the youngster is incarcerated or serving a community sentence.

\section{Discussion and Implications}

The chief purpose of this study is to describe suicide among youth in the juvenile justice systems, including a secured facility and a community service setting. In general, these results suggest an alarming rate of adjudicated youth have seriously considered suicide in the past year; namely, one in five for youth serving community sentences and one out of two for incarcerated youth. Moreover, over $60 \%$ of those youth who have seriously contemplated suicide have also developed a plan, and more than one in three have made an attempt. 
These percentages suggest a rather pressing need for mental health services in the juvenile justice system. These findings have interesting implications for crisis intervention and brief treatment. First of all, the results suggest the likelihood of suicide ideation is greater for incarcerated youth than those in community service. While suicide ideation is still considerable in community settings, the need for assessment and triage is more urgent for incarcerated youth where a routine assessment at intake would help identify what is likely a 50:50 likelihood of a youth who has recently considered suicide.

Moreover, regardless of the setting, these findings suggest that if a youth reports seriously having considered suicide, then in all likelihood he or she has also developed a plan. Thus, these data suggest the initial assessment that identifies a youth with serious suicidal ideation needs to triage him or her for risk based on the availability of a suicide plan and about 35 to $40 \%$ have actually made at least one suicide attempt. Youth in the juvenile justice system seem more likely to have a suicide plan and a good percent will likely have tried, regardless of whether they are incarcerated or serving a community sentence.

These findings are useful in addressing the assessment and triage needs in crisis intervention. First and foremost, these data suggest that if a youth is going to have seriously considered suicide in the past year, then she or he has likely developed a suicide plan, as well. Therefore, knowing a youth has had suicidal thoughts should give immediate rise to the likelihood of a suicide plan. In consideration of the immediacy of the ideation and plan, risk reduction measures may be warranted. Once identified, the course of treatment recommended should be brief treatment. This form of treatment fits the problem because it stabilizes the problem, which is self-transient and circumstantial.

\section{Acknowledgment}

The research was funded by grants from the Oregon Commision on Youth and Families.

\section{References}

Achenbach, T. M. (1991) Manual for the youth selfreport and 1991 profile. Burlington: University of Vermont.

Corcoran, K., \& Fischer, J. (2000). Measures for clinical practice (Vol. 1., pp. 606-609). New York: Free Press.

Hutchinson, J. (1993). Mental health. In L. S. Thompson \& J. A. Farrow (Eds.), Hard time, healing hands: Developing primary health care services for incarcerated youth (pp. 121-133). Arlington, VA: National Center for Education in Maternal and Child Health.

Jobes, D. A., Berman, A. L., \& Martin, C. E. (2000). Adolescent suicidality and crisis intervention. In A. R. Roberts (Ed.), Crisis intervention handbook: Assessment, treatment, and research (2nd ed., pp. 131-151). New York: Oxford University Press.

Litrownik, A. J., Taussig, H. N., Landsverk, J. A., \& Garland, A. F. (1999). Youth entering an emergency shelter care facility: Prior involvement in juvenile justice and mental health systems. Journal of Social Service Research, 25(3), 5-19.

MacKay, A. P., Fingerhut, L. A., \& Duran, C. R. (2000). Adolescent health chartbook. Health, United States. Hyattsville, MD: National Center for Health Statistics.

McCroskey, J. (1988). Policy research: The Los Angeles Roundtable for Children example. Child Welfare, 67(3), 195-204.

Office of Mental Health Services. (1996, September 5). Mental health treatment services for adjudicated delinquent youth: A report to the Legislative Emergency Board. Salem, OR: Office of Mental Health Services and Oregon Youth Authority.

Osman, O., Downs, W. R., Kopper, B. A., Barrios, F. X., Besett, T. M., Linehan, M. M., Baker, M. T., \& Osman, J. R. (1998). The Reason for Living Scale 
for Adolescents (RFL-A): Development and psychometric properties. Journal of Clinical Psychology, 54, 1063-1078.

Prescott, L. (1997). Adolescent girls with co-ocurring disorders in the juvenile justice system. Delmar, NY: GAINS Center.

Stehno, S. M. (1982). Differential treatment of mi- nority children in service systems. Social Work, 27(1), 31-38.

Ware, J. G., Korsinski, M., \& Keller, S. D. (1994). SF-36 physical and mental health summary scales: A user's manual. Boston: The Medical Outcomes Trust. 
